\title{
96. Periods of Hilbert Modular Surfaces
}

\author{
By Takayuki ODA \\ Department of Mathematics, Hokkaido University \\ (Communicated by Kunihiko KodaIRA, M. J. A., Oct. 12, 1981)
}

In this note, we announce some results on the Hodge structures attached to Hilbert modular surfaces. Details will appear elsewhere. As an application of these results, we determine the Picard numbers of some Hilbert modular surfaces.

$\S 1$. Definitions. Let $D$ be a prime number such that $D \equiv 1 \bmod 4$. Suppose that $F=Q(\sqrt{D})$ is a real quadratic field with discriminant $D$ whose class number is one. In this case there exists a unit $\varepsilon$ in the integer ring $\mathcal{O}_{F}$ of $F$ such that its norm $N \varepsilon=-1$.

Let $H \times H$ be the product of two copies of the complex upper half plane $H$. The product $S L_{2}(R) \times S L_{2}(R)$ of the real special linear group $S L_{2}(R)$ of size 2 acts on $H \times H$ factorwise in the usual manner. Composing this action with the natural embedding of $S L_{2}\left(\mathcal{O}_{F}\right)$ into $S L_{2}(R)$ $\times S L_{2}(R)$ by means of the embeddings of $F$ into $R$, we define the action of $S L_{2}\left(\mathcal{O}_{F}\right)$ on $H \times H$.

The quotient surface $S=S L_{2}\left(\mathcal{O}_{F}\right) \backslash(H \times H)$ is a quasiprojective algebraic surface over the complex number field which has a finite number of quotient singularities corresponding to the elliptic fixed points of $S L_{2}\left(\mathcal{O}_{F}\right)$ in $H \times H$ (cf. Hirzebruch [5]).

$\S 2$. The Hodge structure of $H^{2}(S, Q)$. Let $H^{2}(S, \boldsymbol{Q})$ be the second rational cohomology group of the surface $S$. Then, by a theory of Deligne [1], $H^{2}(S, Q)$ has a mixed Hodge structure. Since $S$ has only rational singularities, $S$ is a rational homology manifold, accordingly $W_{1} H^{2}(S, \boldsymbol{Q})=0$ by [1]. Therefore $W_{2} H^{2}(S, \boldsymbol{Q})$ is a homogeneous Hodge structure of weight 2 , which has a polarization

$$
\Psi: W_{2} H^{2}(S, \boldsymbol{Q}) \times W_{2} H^{2}(S, \boldsymbol{Q}) \longrightarrow Q(-2)
$$

induced from the intersection form. We can check that $W_{2} H^{2}(S, Q)$ is equal to the image of the natural mapping $H_{c}^{2}(S, Q) \rightarrow H^{2}(S, Q)$, where $H_{c}^{2}(S, Q)$ is the cohomology with compact supports. We may call this $W_{2} H^{2}(S, Q)$ the Eichler-Shimura cohomology of $S L_{2}\left(\mathcal{O}_{F}\right)$. On the other hand, $W_{2} H^{2}(S, Q)$ coincides with a certain cohomology group of square integrable cocycles, which is studied by Harder [3]. We can check that the Chern forms of the automorphy factors

$$
\eta_{1}=\frac{1}{2 \pi i} \frac{d z_{1} \wedge \overline{d z}_{1}}{y_{1}^{2}}, \quad \eta_{2}=\frac{1}{2 \pi i} \frac{d z_{2} \wedge \overline{d z}_{2}}{y_{2}^{2}}
$$

belong to $W_{2} H^{2}(S, \boldsymbol{Q})$, where $\left(z_{1}, z_{2}\right) \in H \times H, z_{i}=x_{i}+\sqrt{-1} y_{i}(i=1,2)$. 
Let $H_{s p}^{2}(S, \boldsymbol{Q})$ be the orthogonal complement of $\boldsymbol{Q} \eta_{1} \oplus \boldsymbol{Q} \eta_{2}$ in $W_{2} H^{2}(S, Q)$ with respect to $\Psi$. Then $H_{s p}^{2}(S, Q)$ has a homogeneous polarized Hodge structure of weight 2.

Now let us consider the Hecke operators of $S L_{2}\left(\mathcal{O}_{F}\right)$. Since any Hecke operator gives an algebraic correspondence $p_{1} \bigwedge_{S}^{T} \bigwedge_{S}$ of $S$ such that both $p_{1}$ and $p_{2}$ are finite flat, we can define the action of $T$ on $H^{2}(S, Q)$ by $p_{1 *} \circ p_{2}^{*}$. This action of Hecke operators is compatible with the weight filtration and the Hodge filtration. Thus we have the following

Proposition 1 (cf. Hirzebruch [5], Harden [3], and Hida [4]). Let

$$
H_{s p}^{2}(S, Q) \otimes C=H^{2,0} \oplus H^{1,1} \oplus H^{0,2}
$$

be the Hodge decomposition of $H_{s p}^{2}(S, \boldsymbol{Q})$. Then we have natural isomorphism as Hecke ring modules

$$
\begin{aligned}
& H^{2,0} \cong\left\{f\left(z_{1}, z_{2}\right) d z_{1} \wedge d z_{2} \mid f \in S_{2}\left(S L_{2}\left(\mathcal{O}_{F}\right)\right)\right\} \\
& H^{1,1} \cong\left\{G_{\infty}^{*}\left(f\left(z_{1}, z_{2}\right) d z_{1} \wedge d z_{2}\right) \mid f \in S_{2}\left(S L_{2}\left(\mathcal{O}_{F}\right)\right)\right\} \\
& \oplus\left\{H_{\infty}^{*}\left(f\left(z_{2}, z_{2}\right) d z_{1} \wedge d z_{2}\right) \mid f \in S_{2}\left(S L_{2}\left(\mathcal{O}_{F}\right)\right)\right\} \\
& H^{0,2} \cong \bar{H}^{2,0} \cong\left\{G_{\infty}^{*} H_{\infty}^{*}\left(f\left(z_{1}, z_{2}\right) d z_{1} \wedge d z_{2}\right) \mid f \in S_{2}\left(S L_{2}\left(\mathcal{O}_{F}\right)\right)\right\} .
\end{aligned}
$$

Here $S_{2}\left(S L_{2}\left(\mathcal{O}_{F}\right)\right)$ is the space of holomorphic cusp forms of weight 2 with respect to $S L_{2}\left(\mathcal{O}_{F}\right)$, and $G_{\infty}$ and $H_{\infty}$ are involutive automorphism of $S$ induced from

$$
\begin{aligned}
& \tilde{G}_{\infty}=\left(z_{1}, z_{2}\right) \in H \times H \longmapsto \\
& \tilde{H}_{\infty}=\left(z_{1}, z_{2}\right) \in H \times H \longmapsto \\
&\left(z_{1}, \varepsilon^{\prime} \bar{z}_{2}\right) \in H \times H \\
&\left.z_{1}, \varepsilon z_{2}\right) \in H \times H
\end{aligned}
$$

on passing to the quotient, where $\varepsilon$ is a unit satisfying $\varepsilon>0, \varepsilon^{\prime}<0$.

$\S 3$. Hodge structures attached to primitive forms of weight 2. If a Hilbert modular cusp form $f$ with respect to $S L_{2}\left(\mathcal{O}_{F}\right)$ is a common eigenfunction of all Hecke operators, we call it a primitive form. For any primitive form $f$, we denote by $K_{f}$ the field over $\boldsymbol{Q}$ generated by the eigenvalues of $f$. Then $K_{f}$ is a totally real field in our case. Let $f$ be a primitive form, normalized such that its first Fourier coefficient is 1. Then, for any embedding $\sigma: K_{f} \subset C$, we denote by $f^{\sigma}$ the Hilbert modular cusp form obtained from $f$ by applying $\sigma$ to all the Fourier coefficients of $f . \quad f^{\sigma}$ is called a comparison of $f$ (cf. Shimura [7]).

Now let $R_{\boldsymbol{Q}}$ be the $\boldsymbol{Q}$-subalgebra of End $\left(H_{s p}^{2}(S, \boldsymbol{Q})\right)$ generated by the images of Hecke operators. We can show that $R_{Q}$ is a commutative semi-simple algebra. Therefore $R_{Q}$ is isomorphic to a direct sum of fields: $R_{Q}=\oplus_{i=1}^{m} K_{i}$. Let $M$ be the finite set consisting normalized primitive forms of weight 2 with respect to $S L_{2}\left(\Theta_{F}\right)$. We can write $M$ as a disjoint union $M=\cup M_{f}$ of subsets $M_{f}$, so that each $M_{f}$ consists of a normalized primitive form and its companions. The cardinality of $M_{f}$ is the degree $\left[K_{f}: Q\right] . \quad$ For each such subset $M_{f}=\left\{f^{o}\right\}\left(\sigma: K_{f} \longrightarrow C\right)$, we can find a unique subfield $K_{i}$ of $R_{Q}$ such that $K_{i} \simeq K_{f}$ by Multiplicity 
One Theorem. Letting $e_{i}$ be the primitive idempotent of $R_{Q}$ corresponding to the factor $K_{i}$ we put

$$
H^{2}\left(M_{f}, \boldsymbol{Q}\right)=e_{i} H_{s p}^{2}(S, \boldsymbol{Q}) \text {. }
$$

$H^{2}\left(M_{f}, \boldsymbol{Q}\right)$ is a sub-Hodge structure of $H_{s p}^{2}(S, \boldsymbol{Q})$. Restricting the action of $R_{Q}$, we can define an action of each $K_{i}$, accordingly an action

$$
\theta_{f}^{*}: K_{f} \longrightarrow \text { End }\left(H^{2}\left(M_{f}, \boldsymbol{Q}\right)\right)
$$

of $K_{f}$ via the identification $K_{i} \widetilde{\rightarrow} K_{f}$. Thus we have a direct sum decomposition

$$
H_{s p}^{2}(S, \boldsymbol{Q})=\oplus f^{\prime} \in \Xi H^{2}\left(M_{f}, \boldsymbol{Q}\right),
$$

where $\Xi$ is a subset of $M$, so that $M=\cup_{f \in s} M_{f}$ (disjoint). Let

$$
\Psi_{f}: H^{2}\left(M_{f}, \boldsymbol{Q}\right) \times H^{2}\left(M_{f}, \boldsymbol{Q}\right) \longrightarrow \boldsymbol{Q}(-2)
$$

be the polarization obtained by restricting $\Psi$ to $H^{2}\left(M_{f}, \boldsymbol{Q}\right)$. We can show there exists a $K_{f}$-bilinear form

$$
\psi_{f}: H^{2}\left(M_{f}, \boldsymbol{Q}\right) \times H^{2}\left(M_{f}, \boldsymbol{Q}\right) \longrightarrow K_{f}
$$

so that $\Psi_{f}=\operatorname{tr}_{K_{f / Q}}\left(\psi_{f}\right)$. We obtain the following

Theorem. (i ) $H^{2}\left(M_{f}, Q\right)$ is a rational polarized Hodge structure of weight 2, on which $K_{f}$ acts as endomorphisms of Hodge structure via

The polarization

$$
\theta_{f}^{*}: K_{f} \prec \operatorname{End}\left(H^{2}\left(M_{f}, \boldsymbol{Q}\right)\right)
$$

$$
\Psi_{f}: H^{2}\left(M_{f}, \boldsymbol{Q}\right) \times H^{2}\left(M_{f}, \boldsymbol{Q}\right) \longrightarrow \boldsymbol{Q}(-2)
$$

is written as $\Psi_{f}=t r_{K_{f / Q}}\left(\psi_{f}\right)$ by a $K_{f}$-bilinear non-degenerate symmetric form

$$
\psi_{f}: H^{2}\left(M_{f}, \boldsymbol{Q}\right) \times H^{2}\left(M_{f}, \boldsymbol{Q}\right) \longrightarrow K_{f} .
$$

(ii) For any embedding $\sigma: K_{f} \longrightarrow R$ of the totally real number field $K_{f}$, the scalar extension $\psi_{f} \otimes_{K_{f}, \sigma} R$ has signature $(2,2)$. For any embedding $\sigma: K_{f} \longrightarrow C, H^{2}\left(M_{f}, \boldsymbol{Q}\right) \otimes_{K_{f}, \sigma} C$ has a Hodge structure of weight 2 with Hodge number $\left\{h^{2,0}=h^{0,2}=1, h^{1,1}=2\right\}$.

§4. Main theorem A. Main Theorem A. Let $f$ be a primitive form of weight 2 with respect to $S L_{2}\left(\Theta_{F}\right)$. Then we can find two isogeny classes $\boldsymbol{A}_{f}^{(1)} \otimes \boldsymbol{Q}, \boldsymbol{A}_{f}^{(2)} \otimes \boldsymbol{Q}$ of abelian varieties $\boldsymbol{A}_{f}^{(1)}, \boldsymbol{A}_{f}^{(2)}$ over $\boldsymbol{C}$ of dimension $d=\left[K_{f}: Q\right]$ with endomorphisms

$$
\theta^{(i) *}: K_{f} \longrightarrow \operatorname{End}\left(A_{f}^{(i)}\right) \otimes_{Z} \boldsymbol{Q} \quad(i=1,2),
$$

such that there exists an isomorphism of Hodge structures

$$
H^{2}\left(M_{f}, \boldsymbol{Q}\right) \stackrel{\sim}{\longrightarrow} H^{1}\left(A_{f}^{(1)}, \boldsymbol{Q}\right) \otimes_{K_{f}} H^{1}\left(A_{f}^{(2)}, \boldsymbol{Q}\right)
$$

compatible with the actions of $K_{f}$.

Corollary 1. Let $H_{s p}^{2}(S, \boldsymbol{Q})_{\mathrm{alg}}$ be the sub-Hodge structure of $H_{s p}^{2}(S, \boldsymbol{Q})$ generated by algebraic cycles and let $H^{2}\left(M_{f}, \boldsymbol{Q}\right)_{\mathrm{alg}}$ be its projection to $H^{2}\left(M_{f}, Q\right)$. Then we have an isomorphism of $K_{f}$-modules

$$
H^{2}\left(M_{f}, \boldsymbol{Q}\right)_{\mathrm{alg}} \cong \operatorname{Hom}_{\mathcal{O}_{f}}\left(A_{f}^{(1)}, A_{f}^{(2)}\right) \otimes_{Z} \boldsymbol{Q} .
$$

Here $\mathcal{O}_{f}$ is a sufficiently small order of $K_{f}$ so that $\theta^{(i) *}\left(\mathcal{O}_{f}\right) \longrightarrow \operatorname{End}\left(A_{f}^{(i)}\right)$ for both $i=1$, and $i=2$. 
Corollary 2. We have the following equivalences

(i) $\operatorname{rank}_{K_{f}} H^{2}\left(M_{f}, Q\right)_{\text {alg }}=0 . \Leftrightarrow$ There is no isogeny between $A_{f}^{(1)}$ and $A_{f}^{(2)}$ compatible with the action of $K_{f}$.

(ii) $\operatorname{rank}_{K_{f}} H^{2}\left(M_{f}, Q\right)_{\text {alg }}=1$. $\Leftrightarrow$ There exists an isogeny $\phi: A_{f}^{(1)} \rightarrow A_{f}^{(2)}$ compatible with the action of $K_{f}$, but neither $A_{f}^{(1)}$ nor $A_{f}^{(2)}$ is a CM-type abelian variety with a $C M$-field $L$ satisfying $\left[L: K_{f}\right]=2$.

(iii) $\operatorname{rank}_{K f} H^{2}\left(M_{f}, Q\right)_{\mathrm{alg}}=2 . \Leftrightarrow$ Both $A_{f}^{(1)}$ and $A_{f}^{(2)}$ are $C M$-type abelian varieties with the same $C M$-field $L$ with $\left[L: K_{f}\right]=2$ and there exists an isogeny $\phi: A_{f}^{(1)} \rightarrow A_{f}^{(2)}$ compatible with the action of $L$.

$\S 5$. Lifting and Hodge structures (Main theorem B). Let $S_{2}\left(\Gamma_{0}(D),(D / *)\right)$ be the space of real Neben type elliptic modular cusp forms of weight 2 with respect to $\Gamma_{0}(D)$ and with multiplicator $(D / *)$. Then there is a correspondence of primitive forms $h$ of $S_{2}\left(\Gamma_{0}(D),(D / *)\right)$ to primitive form of $S_{2}\left(S L_{2}\left(\mathcal{O}_{F}\right)\right.$ ) constructed by Naganuma [6] (cf. also Doi-Naganuma [2], Zagier [9]). On the other hand we can attach abelian variety $A_{h}$ of dimension $\left[K_{h}: Q\right]$ to each primitive form $h$ $\in S_{2}\left(\Gamma_{0}(D),(D / *)\right)$ by the theory of Shimura [7], where $K_{h}$ is $C M$-field which is the field of eigenvalues of $h$. Let $\eta=\left(\begin{array}{lr}0 & -1 \\ D & 0\end{array}\right)$ be the main involution of $\Gamma_{0}(D)$ and put $B_{h}=(1+\eta) A_{h}$. Then it is known that $A_{h}$ is isogenous to the product $B_{h} \times B_{h}$, and by restriction the maximal totally real subfield $k_{h}=\left\{a \in K_{h} \mid a=a^{\rho}\right\}$ acts on $B_{h}$ as endomorphisms, where $\rho$ is the complex conjugation.

Main Theorem B. Let $h$ be a primitive form of $S_{2}\left(\Gamma_{0}(D),(D / *)\right)$, and let $f$ be a primitive form of $S_{2}\left(S L_{2}\left(\mathcal{O}_{F}\right)\right)$ corresponding to $h$ by Naganuma construction ([6]). Then $k_{h}=K_{f}$ and we have isogenies of abelian varieties

$$
\phi_{i}: B_{h} \longrightarrow A_{f}^{(i)} \quad(i=1,2)
$$

compatible with the action of $k_{h}=K_{f}$.

Combining this result with a result of Shimura [7], which tells that $B_{h}$ has no complex multiplication, by Main Theorem A we can show the following

Main Theorem C. If a primitive form $f \in S_{2}\left(S L_{2}\left(\mathcal{O}_{F}\right)\right)$ is obtained from a primitive form $h$ by Naganuma construction, then

$$
\operatorname{rank}_{K_{f}} H^{2}\left(M_{f}, \boldsymbol{Q}\right)_{\mathrm{alg}}=1 \text {. }
$$

§6. An application. By Theorem $\mathrm{C}$, we can determine completely the Picard numbers of some Hilbert modular surfaces.

Theorem. Assume that $D<193$ or $D=197,269,293,317$. Let $\tilde{S}$ be a complete smooth model of Hilbert modular surface $S$, let $\rho(\tilde{S})$ be the Picard number of $\tilde{S}$, i.e. the rank over $\boldsymbol{Q}$ of the subspace of $H^{2}(\tilde{S}, \boldsymbol{Q})$ generated by algebraic cycles, and let $b_{2}(\tilde{S})$ be the second Betti number of $\tilde{S}$. Then the birational invariant $b_{2}(\tilde{S})-\rho(\tilde{S})$ is equal to $3 \cdot p_{g}(\tilde{S})$, where $p_{g}(\tilde{S})$ is the geometric genus of $\tilde{S}$. 
$\S 7$. Examples on $\ell$-adic cohomology groups. The surface $S$ has a canonical model defined over $\boldsymbol{Q}$ ([10]). Let us denote this model over $\boldsymbol{Q}$ by the same symbol $S$. Now consider the $b$-adic cohomology group $H_{e t}^{2}\left(S \times \overline{\boldsymbol{Q}}, \boldsymbol{Q}_{\ell}\right)$. Since $\eta_{1}$ and $\eta_{2}$ in $W_{2} H_{e t}^{2}\left(S \times \overline{\boldsymbol{Q}}, \boldsymbol{Q}_{\ell}\right)$ are the Chern classes of the line bundles corresponding to the two automorphy factors, we have a natural Gal $(\bar{F} / F)$-module $H_{s p}^{2}\left(S \times \overline{\boldsymbol{Q}}, \boldsymbol{Q}_{\ell}\right)$ so that

$$
H_{s p}^{2}\left(S \times \overline{\boldsymbol{Q}}, \boldsymbol{Q}_{\ell}\right) \cong H_{s p}^{2}(S \times \boldsymbol{C}, \boldsymbol{Q}) \otimes \boldsymbol{Q}_{\ell}
$$

by the comparison theorem of Artin. Combining the results of [11] with Main Theorem B, we have the following

Theorem. Assume that $D=29,37$ or 41 . Then for a sufficiently large algebraic extension $L$ of $\boldsymbol{F}$, we have an isomorphism of $\operatorname{Gal}(\overline{\mathbf{Q}} / L)$ modules

$$
H_{s p}^{2}\left(S \times \overline{\boldsymbol{Q}}, \boldsymbol{Q}_{\ell}\right) \cong H^{1}\left(B_{n} \times \overline{\boldsymbol{Q}}, \boldsymbol{Q}_{\ell}\right) \otimes_{\boldsymbol{Q}_{\ell}} H^{1}\left(B_{h} \times \overline{\boldsymbol{Q}}, \boldsymbol{Q}_{\ell}\right) .
$$

Here $B_{h}$ is the elliptic curve attached to the primitive forms $h$ and $h$ in $S_{2}\left(\Gamma_{0}(D),(D / *)\right.$ ) (note that $\left.\operatorname{dim}_{C} S_{2}\left(\Gamma_{0}(D),(D / *)\right)=2\right)$.

\section{References}

[1] Deligne, P.: Theorie de Hodge. II; III. Pub. Math. Inst. Hautes Etudes Sci., 40, 5-58 (1971) ; 44, 5-77 (1974).

[2] Doi, K., and Naganuma, H.: On the functional equation of certain Dirichlet series. Invent. math., 9, 1-14 (1969).

[3] Harder, G.: On the cohomology of $S L_{2}(\mathcal{O})$. Lie Groups and their Representations. Proc. of the summer school on group representations, pp. 139150 (1975).

[4] Hida, H.: On the abelian varieties with complex multiplication as factors of the abelian variety attached to Hilbert modular forms. Japan J. Math., 5, 157-208 (1979).

[5] Hirzebruch, F.: Hilbert modular surfaces. L'Ens. Math., 19, 183-281 (1973).

[6] Naganuma, H.: On the coincidence of two Dirichlet series associated with cusp forms of Hecke's "Neben"-type and Hilbert modular forms over real quadratic field. J. Math. Soc. Japan, 25, 547-555 (1973).

[7] Shimura, G.: Introduction to the Arithmetical Theory of Automorphic Functions. Iwanami Shoten and Princeton Univ. Press (1971).

[8] — : Class fields over real quadratic fields and Hecke operators. Ann. of Math., 95, 130-190 (1972).

[9] Zagier, D.: Modular forms associated to real quadratic fields. Invent. math., 30, 1-46 (1975).

[10] Shimura, G.: On canonical models of arithmetic quotients of bounded symmetric domains. I; II. Ann. of Math., 91, 114-222; 92, 528-549 (1970).

[11] Deligne, P.: La conjecture de Weil pour les surfaces K3. Invent. math., 15, 206-226 (1972). 